\title{
Probabilistic Constrained Approach for Distributed Robust Beamforming Design in Cognitive Two-way Relay Networks
}

\author{
Xueyan Chen", Li Guo", Chao Dong ${ }^{1}$, Jiaru Lin ${ }^{1}$, Xingwang Li $^{2}$ and Charles C. Cavalcante ${ }^{3}$ \\ ${ }^{1}$ Key Lab of Universal Wireless Communications, Ministry of Education, Beijing University of Posts and \\ Telecommunications, Beijing, China \\ ${ }^{2}$ School of Physics and Electronic Information Engineering, Henan Polytechnic University, Jiaozuo, China \\ ${ }^{3}$ Wireless Telecommunications Research Group, Federal University of Ceará, Campus do Pici, Bl. 722, ZIP \\ 60455-760, Fortaleza-CE, Brazil \\ [e-mail: chenxybupt@163.com, guoli@bupt.edu.cn, dongchao@bupt.edu.cn, jrlin@bupt.edu.cn, \\ lixingwangbupt@gmail.com, charles@gtel.ufc.br] \\ *Corresponding author: Li Guo
}

Received February 28, 2017; revised June 16, 2017; accepted September 17, 2017; published January 31, 2018

\begin{abstract}
In this paper, we propose the distributed robust beamforming design scheme in cognitive two-way amplify-and-forward (AF) relay networks with imperfect channel state information (CSI). Assuming the CSI errors follow a complex Gaussian distribution, the objective of this paper is to design the robust beamformer which minimizes the total transmit power of the collaborative relays. This design will guarantee the outage probability of signal-tointerference-plus-noise ratio (SINR) beyond a target level at each secondary user (SU), and satisfies the outage probability of interference generated on the primary user (PU) above the predetermined maximum tolerable interference power. Due to the multiple CSI uncertainties in the two-way transmission, the probabilistic constrained optimization problem is intractable and difficult to obtain a closed-form solution. To deal with this, we reformulate the problem to the standard form through a series of matrix transformations. We then accomplish the problem by using the probabilistic approach based on two sorts of Bernstein-type inequalities and the worst-case approach based on S-Procedure. The simulation results indicate that the robust beamforming designs based on the probabilistic method and the worst-case method are both robust to the CSI errors. Meanwhile, the probabilistic method can provide higher feasibility rate and consumes less power.
\end{abstract}

Keywords: cognitive network, beamforming, two-way relay, Bernstein-type inequality, semidefinite relaxation

This work is supported in part by the National Natural Science Foundation of China (61271178), in part by the Fundamental Research Funds for the Central Universities (BUPT No.2015RC06), in part by the National Nature Science Foundation of China (61631003), and in part by the Henan Scientific and Technological Research Project under Grant 172102210023. 


\section{Introduction}

Cognitive radio (CR) is an intelligent wireless communication technology developed from software radio (SR), which has been proposed by J. Mitola in 1999. It is a promising way to increase the efficiency of spectrum utilization in wireless systems and networks [1]. In the CR network, unlicensed secondary users (SUs) are allowed to access the spectrum which has been allocated to the licensed primary users (PUs). There are three main paradigms for the SUs to access the spectrum, namely interweave, overlay and underlay [2]. In recent years, along with the development of collaborative relay beamforming (CRBF) technology, it has been used to extend the range of wireless communications and mitigate the channel fading in wireless networks [3-5]. Meanwhile, the two-way relaying technology can provide better spectrum usage compared to the one-way relaying scheme, for the reason that the two-way relay technology enables two users communicate in both directions simultaneously to exchange information with each other $[6,7]$.

Currently, a considerable number of research efforts have been dedicated to CR systems, which were usually based on the assumption of perfect channel state information (CSI) [8-10]. However, in practice, the CSI errors are inevitable due to the estimation errors, quantization errors and feedback errors. In [11], the authors have analyzed the uncertainty about the channel coefficients would cause performance loss on the network. The effects of imperfect channel knowledge on the SU capacity in CR networks were analysed in [12, 13]. In order to overcome the effects of the CSI errors in communication, the robust beamforming design is required when the CSI is imperfect. According to the model of CSI errors, robust beamforming design can be divided into two different classes. The first one is the worst-case assumption in which the CSI errors distribution cannot be acquired exactly, and the errors are deterministic and bounded in a region [14-16]. However, this method only considered the worst situation which rarely occur in practical communications, thus bringing conservative results. The second one is an statistical assumption, in which the CSI errors follow a known statistical distribution.

Most of the prior works about robust beamforming designs only consider one-hop transmission without relays [17-20]. In [17], a transmit beamforming design for MIMO communications that maximizes the average SNR and guarantees robustness against channel estimation errors was investigated. A probabilistic robust beamforming design problem under a single-cell system with multiple-antenna transmitter and $K$ single-antenna receivers was studied in $[18,19]$. Nevertheless, only a few works concentrate on the cognitive network as the CSI errors exist in all channels estimation [20]. In addition, the authors investigated the unified transceiver designs framework for different wireless systems in [21, 22], and transceiver designs for dual-hop and multi-hop MIMO relaying network were discussed with perfect and imperfect CSI, while they did not tackle the problem from the probabilistic aspect of the constraints. The probability constrained robust beamforming designs which consider two-hop transmission with relays in conventional wireless network have been investigated in [23-25]. To the best of our knowledge, the robust beamforming design for cognitive two-way relay networks with statistical distribution model of CSI errors has not been investigated yet.

Motivated with the above facts, we analyze the distributed robust beamforming design with two probability constrained in cognitive two-way relay networks. The objective of this paper is minimizing the transmit power of the collaborative relays, while guaranteeing probabilistic constraint of target SINR at each SU above the target value, and satisfying 
probabilistic constraint of interference power at primary user (PU) below the given threshold. The main contributions of this work are summarized as follows:

- We assume that the channel gains during multiple access (MAC) phase and broadcast (BC) phase are reciprocal and uncertain, the channel errors follow a known independent and identically distributed complex Gaussian random distribution. Under this assumption, we formulate the problem of distributed robust beamforming design with probabilistic constraints for cognitive two-way relay networks, which has not been well investigated before.

- After reformulating the probabilistic constraints to the tractable forms through a series of matrix transformations, we propose the probabilistic method based on two kinds of Bernstein-type inequalities and rank-one relaxtion to convert the original non-convex problem to the standard convex problem, then we can derive the solution for the original problem.

- We also propose the robust beamforming design by worst-case method based on extended S-procedure and rank-one relaxtion to tackle the problem. We verify that both probabilistic method and the worst-case method can overcome the effects of CSI errors, In addition, the probabilistic method can provide higher feasibility rate and is more energy-saving.

Throughout this paper, the key mathematical notations are used as follows: boldface lowercase and uppercase letters denote vectors and matrices, respectively. $(\cdot)^{T},(\cdot)^{H}, \operatorname{Tr}(\cdot)$, $\|\cdot\|$ and $\operatorname{rank}(\cdot)$ and $\operatorname{BD}(\cdot)$ denote transpose, conjugate transpose, the trace, the Frobenius norm, rank and block diagonalization of matrix, respectively. $\mathbf{1}$ denotes the vector with all elements being 1 , $\mathbf{I}$ denotes the identity matrix. $\mathbf{A} \circ \mathbf{B}$ denotes Hadamard product of matrix $\mathbf{A}$ and matrix $\mathbf{B}, \operatorname{Re}\{\cdot\}$ represents the real part of the associated number, $\operatorname{Pr}\{\cdot\}$ is the probability operator. $\mathbf{X} \geq 0$ indicates that $\mathbf{X}$ is a positive semidefinite matrix. $C^{K}$ denotes the space of $K \times 1$ matrices with complex entries, $\mathbf{H}^{K}$ is the set of $K \times K$ complex Hermitian matrices. $\operatorname{diag}(\mathbf{x})$ returns a diagonal matrix with diagonal elements equal to vector $\mathbf{x}$, $\operatorname{diag}(\mathbf{A})$ returns the diagonal elements of the matrix $\mathbf{A}, \operatorname{vec}(\mathbf{A})$ means the vectorization of matrix $\mathbf{A}$ by stack its columns into a vector. $\mathbf{x} \sim \mathcal{C N}(\mathbf{m}, \mathbf{V})$ means that vector $\mathbf{x}$ is complex Gaussian distributed with mean vector $\mathbf{m}$ and covariance matrix $\mathbf{V}$.

The rest of paper is organized as follows. In Sect. 2 we describe the system model of our optimization problem. Both robust beamforming design based on probabilistic method by using Bernstein-Type inequalities and robust beamforming design based on worst-case method by using S-Procedure are proposed in Sect. 3. Finally, the simulation results and conclusions are given in Sects. 4 and 5, respectively.

\section{System Model}

The underlay two-way relay CR network is shown in Fig. 1, which is composed of a primary user (PU), a pair of secondary transceiver nodes, i.e. $S_{1}$ and $S_{2}$, and $K$ collaborative relays. Each communication node is equipped with a single antenna. It is assumed that there is no direct link between $S_{1}$ and $S_{2}$, i.e. they communicate with each other through $K$ random distributed collaborative amplify-and-forward (AF) relays in the secondary network. We 
assume that the secondary network is allowed to share the same spectrum with primary network in an underlay manner. The bidirectional communication takes two phases, the MAC phase and the $\mathrm{BC}$ phase. The channel gains during the MAC phase and $\mathrm{BC}$ phase are reciprocal, i.e. the information transmits in time-division-duplex (TDD) systems.

In the MAC phase, the two SUs transmit their signal meant for each other to the relays simultaneously, and the relays receive the signal transmitted from PU at the same time, accordingly, the received signal at the relays is

$$
\mathbf{y}_{R}=\mathbf{h}_{1} x_{1}+\mathbf{h}_{2} x_{2}+\mathbf{h}_{0} x_{0}+\mathbf{n}_{R}
$$

where $x_{1} \in C$ and $x_{2} \in C$ are the transmit signals from $S_{1}$ and $\mathbf{S}_{2}$, respectively, and $\mathrm{E}\left[\left|x_{i}\right|^{2}\right]=P_{i}, i \in\{1,2\}, x_{0} \in C$ denotes the signal transmitted from $\mathrm{PU}$, and $\mathrm{E}\left[\left|\boldsymbol{x}_{\mathbf{0}}\right|^{2}\right]=\boldsymbol{P}_{\mathbf{0}}$. The signals $\boldsymbol{x}_{\mathbf{0}}, \boldsymbol{x}_{\mathbf{1}}$ and $\boldsymbol{x}_{2}$ are assumed to be independent among each other. The vector $\mathbf{n}_{R}=\left[n_{R 1}, n_{R 2}, \cdots, n_{R K}\right]^{T}$, where $n_{R k}$ is the additive noise at the $k$ th relay $\mathbf{R}_{k}$, and $n_{R k} \sim \mathcal{C N}\left(0, \sigma_{R}^{2}\right) . \mathbf{h}_{i}=\left[h_{i 1}, h_{i 2}, \cdots, h_{i K}\right]^{T}, i \in\{1,2\}, h_{i k}$ is the complex-valued channel coefficient from $\mathbf{S}_{i}$ to the $k$ th relay $\mathbf{R}_{k} . \mathbf{h}_{0}=\left[h_{01}, h_{02}, \cdots, h_{0 K}\right]^{T}, h_{0 K}$ is the complex-valued channel coefficient from PU to the $k$ th relay $\mathbf{R}_{k}$. The vector $\mathbf{y}_{R}=\left[y_{R 1}, y_{R 2}, \cdots, y_{R K}\right]^{T}$, where $\boldsymbol{y}_{\boldsymbol{R} k}$ is the received signal at $\mathbf{R}_{\boldsymbol{k}}$.

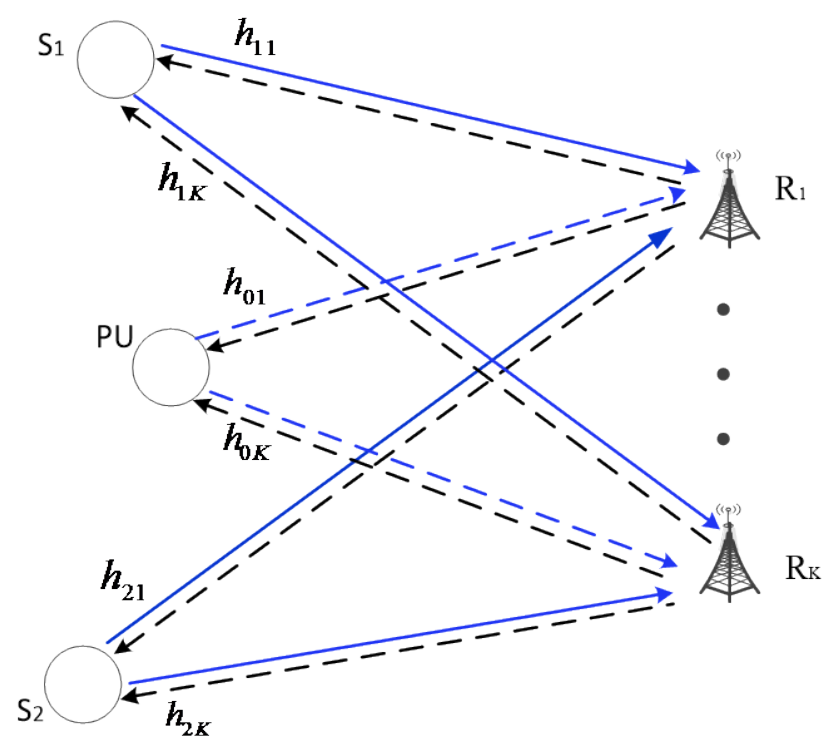

Fig. 1. System model. The blue lines represent the MAC phase, the black lines represent the BC phase. The solid arrow lines are signal streams, the dashed arrow lines are interference streams.

In the BC phase, the relay nodes broadcast the received signal in the MAC phase after multiplies by a complex relay beamformer $\mathbf{w} \in C^{K}$ to SUs and PU simultaneously. Thus the broadcast processed signal at the relay nodes can be written as 


$$
\begin{aligned}
\mathbf{x}_{R} & =\mathbf{W} \mathbf{y}_{R} \\
& =\mathbf{W h}_{1} x_{1}+\mathbf{W h} \mathbf{h}_{2} x_{2}+\mathbf{W h} \mathbf{h}_{0} x_{0}+\mathbf{W} \mathbf{n}_{R},
\end{aligned}
$$

where $\mathbf{x}_{R}=\left[x_{R 1}, x_{R 2}, \cdots, x_{R K}\right]^{T}, \mathbf{W}=\operatorname{diag}(\mathbf{w})$, and $\mathbf{w}=\left[w_{1}, w_{2}, \cdots, w_{K}\right]^{T}$.

The received signal at $S_{i}, i \in\{1,2\}$, can be denoted as

$$
\begin{aligned}
y_{i} & =\mathbf{h}_{i}^{T} \mathbf{x}_{R}+n_{i} \\
& =\underbrace{\mathbf{h}_{i}^{T} \mathbf{W} \mathbf{h}_{\bar{i}} x_{\bar{i}}}_{\text {desired signal }}+\underbrace{\mathbf{h}_{i}^{T} \mathbf{W} \mathbf{h}_{i} x_{i}+\mathbf{h}_{i}^{T} \mathbf{W} \mathbf{h}_{0} x_{0}}_{\text {interference }}+\underbrace{\mathbf{h}_{i}^{T} \mathbf{W} \mathbf{n}_{R}+n_{i}}_{\text {noise }}, \forall i \in\{1,2\},
\end{aligned}
$$

where $n_{i}$ is the additive noise at $\mathrm{S}_{i}$, it is assumed that $n_{i} \sim \mathcal{C N}\left(0, \sigma_{i}^{2}\right)$, and $i \in\{1,2\}, \overline{1}=2$, $\overline{2}=1$, then $\mathbf{h}_{\overline{1}}=\mathbf{h}_{2}$ and $\mathbf{h}_{\overline{2}}=\mathbf{h}_{1}$.

The interference signal at PU caused by the relays is given as

$$
\begin{aligned}
y_{I} & =\mathbf{h}_{0}^{T} \mathbf{x}_{R} \\
& =\mathbf{h}_{0}^{T} \mathbf{W} \mathbf{h}_{1} x_{1}+\mathbf{h}_{0}^{T} \mathbf{W} \mathbf{h}_{2} x_{2}+\mathbf{h}_{0}^{T} \mathbf{W} \mathbf{h}_{0} x_{0}+\mathbf{h}_{0}^{T} \mathbf{W} \mathbf{n}_{R} .
\end{aligned}
$$

During the MAC phase, the channel vector can be estimated by a central processor which is embedded in (or placed near to) the relay nodes. In the BC phase, the channel vector can be measured by the central processor at the SUs and PU, then sended to the relays via feedback channel. Considering limited training at SUs, less cooperation between cognitive relay nodes and PU, estimation errors, quantization errors and feedback errors, we assume the CSI errors existed during the both phases in our model. Then we adopt a circular complex Gaussian channel error model [20], the CSI error model is given by

$$
\mathbf{h}_{i}=\hat{\mathbf{h}}_{i}+\mathbf{e}_{i}, \quad \forall i \in\{0,1,2\},
$$

where $\hat{\mathbf{h}}_{i}$ is the estimated CSI vector, $\mathbf{e}_{i}$ is the corresponding independent CSI error vector with $\mathbf{e}_{i} \sim \mathcal{C N}\left(\mathbf{0}, \mathbf{E}_{i}\right)$, where $\mathbf{E}_{i}$ is the covariance of the CSI error vector. We assume that $\mathbf{E}_{i}=\varepsilon \mathbf{I}_{K}(\varepsilon>0)$ for simplicity sake [20]. as

Accordingly, the total transmit power of the relay nodes in the BC phase can be expressed

$$
P_{R}=\sum_{i=0}^{2} P_{i}\left\|\mathbf{W}\left(\hat{\mathbf{h}}_{i}+\mathbf{e}_{i}\right)\right\|^{2}+\sigma_{R}^{2}\|\mathbf{W}\|^{2},
$$

The received signal at $S_{i}$ by considering the CSI errors can be represented as 


$$
\begin{aligned}
\bar{y}_{i}= & \underbrace{\mathbf{h}_{i}{ }^{T} \mathbf{W} \mathbf{h}_{\bar{i}} x_{\bar{i}}}_{\text {desired signal }}+\underbrace{\hat{\mathbf{h}}_{i}{ }^{T} \mathbf{W} \hat{\mathbf{h}}_{i} x_{i}}_{\text {self-interference }}+\underbrace{\mathbf{h}_{i}^{T} \mathbf{W} \mathbf{n}_{R}+n_{i}}_{\text {noise }} \\
& +\underbrace{\hat{\mathbf{h}}_{i}^{T} \mathbf{W} \mathbf{e}_{i} x_{i}+\mathbf{e}_{i}^{T} \mathbf{W} \mathbf{h}_{i} x_{i}+\mathbf{h}_{i}{ }^{T} \mathbf{W} \mathbf{h}_{0} x_{0}}_{\text {interference }} \quad, \quad i \in\{1,2\}
\end{aligned}
$$

Since $S_{i}$ knows its own transmitted signal, the self-interference can be completely canceled from Eq. (7) with the known estimated channel information. Then the desired signal power at $S_{i}$ yields as

$$
P_{s}^{i}=P_{\bar{i}}\left\|\left(\hat{\mathbf{h}}_{i}+\mathbf{e}_{i}\right)^{T} \mathbf{W}\left(\hat{\mathbf{h}}_{\bar{i}}+\mathbf{e}_{\bar{i}}\right)\right\|^{2},
$$

The interference power and noise power at $S_{i}$ can be denoted as $P_{\text {int }}{ }^{i}$ and $P_{n}{ }^{i}$, respectively, and given as

$$
\begin{gathered}
P_{\mathrm{int}}{ }^{i}=P_{i}\left\|\hat{\mathbf{h}}_{i}{ }^{T} \mathbf{W} \mathbf{e}_{i}+\mathbf{e}_{i}^{T} \mathbf{W}\left(\hat{\mathbf{h}}_{i}+\mathbf{e}_{i}\right)\right\|^{2}+P_{0}\left\|\left(\hat{\mathbf{h}}_{i}+\mathbf{e}_{i}\right)^{T} \mathbf{W}\left(\hat{\mathbf{h}}_{0}+\mathbf{e}_{0}\right)\right\|^{2}, \\
P_{n}^{i}=\sigma_{R}{ }^{2}\left\|\left(\hat{\mathbf{h}}_{i}+\mathbf{e}_{i}\right)^{T} \mathbf{W}\right\|^{2}+\sigma_{i}{ }^{2} .
\end{gathered}
$$

Therefore, the SINR at $S_{i}$ yields as

$$
\operatorname{SINR}_{i}=\frac{P_{s}^{i}}{P_{\mathrm{int}}^{i}+P_{n}^{i}}, \quad \forall i \in\{1,2\}
$$

The interference power at PU caused by the relays is then given by the following:

$$
P_{I}=\sum_{i=0}^{2} P_{i}\left\|\left(\hat{\mathbf{h}}_{0}+\mathbf{e}_{0}\right)^{T} \mathbf{W}\left(\hat{\mathbf{h}}_{i}+\mathbf{e}_{i}\right)\right\|^{2}+\sigma_{R}^{2}\left\|\left(\hat{\mathbf{h}}_{0}+\mathbf{e}_{0}\right)^{T} \mathbf{W}\right\|^{2} .
$$

\section{Robust Beamforming Design}

The objective of this paper is to design the robust beamforming vector which minimizes the total transmit power of the relays in the collaborative two-way relay CR network, while subject to the probabilistic constraints of the SINR at the two SUs above the target value, as well as the probabilistic constraint of the interference at PU below a given threshold. Considering the CSI errors in the expression of $P_{R}$, the probabilistic constrained robust beamforming design can be formulated as following Problem 1:

$$
\min _{\mathbf{w}, t} t
$$




$$
\begin{array}{ll}
\text { s.t. } & \operatorname{Pr}\left\{P_{R} \leq t\right\} \leq \rho_{R} \\
& \operatorname{Pr}\left\{\operatorname{SINR}_{i} \geq \gamma_{i}\right\} \geq 1-\rho_{i} \quad\{i=1,2\} \\
& \operatorname{Pr}\left\{P_{I} \geq \zeta\right\} \leq \rho_{0},
\end{array}
$$

where $\gamma_{i}$ is the target SINR threshold at $S_{i}$, and $\zeta$ denotes the maximum tolerable interference power at PU. $\rho_{i} \in(0,1], \rho_{0} \in(0,1]$ and $\rho_{R} \in(0,1]$ are the maximum outage probability at $S_{i}$, PU and relays, respectively.

Since the probabilistic constraints in Eq. (13b), Eq. (13c) and Eq. (13d) contain quadratic and higher forms of complex Gaussian random variables, it is difficult to derive the closed-form solution of Problem 1. In order to solve such intractable problem, we replace the probabilistic constraints with their approximate forms. We define the following quantities:

$$
\begin{aligned}
& \mathbf{u}=\operatorname{vec}(\mathbf{W}), \mathbf{S}=\mathbf{u u}^{H}, \\
& \mathbf{E}=\mathbf{I}_{K^{2} \times K^{2}}, \mathbf{A}_{0}=\left[\begin{array}{lll}
\mathbf{I}_{K \times K} & \mathbf{0}_{K \times K} & \mathbf{0}_{K \times K}
\end{array}\right], \\
& \mathbf{A}_{1}=\left[\begin{array}{lll}
\mathbf{0}_{K \times K} & \mathbf{I}_{K \times K} & \mathbf{0}_{K \times K}
\end{array}\right], \mathbf{A}_{2}=\left[\begin{array}{lll}
\mathbf{0}_{K \times K} & \mathbf{0}_{K \times K} & \mathbf{I}_{K \times K}
\end{array}\right], \\
& \mathbf{B}_{1}=\mathbf{1}_{K \times 1} \otimes \mathbf{I}_{K \times K}, \mathbf{B}_{2}=\mathbf{I}_{K \times K} \otimes \mathbf{1}_{K \times 1} \text {, } \\
& \mathbf{C}_{i}=\operatorname{diag}\left(\mathbf{1}_{K \times 1} \otimes \hat{\mathbf{h}}_{i}\right), \mathbf{D}_{i}=\operatorname{diag}\left(\hat{\mathbf{h}}_{i} \otimes \mathbf{1}_{K \times 1}\right), \\
& \mathbf{G}_{i j}=\mathbf{B}_{j} \mathbf{A}_{i}, \mathbf{g}_{i j}=\left(\hat{\mathbf{h}}_{j} \otimes \mathbf{1}_{K \times 1}\right) \circ\left(\mathbf{1}_{K \times 1} \otimes \hat{\mathbf{h}}_{i}\right), \\
& \mathbf{h}=\left[\begin{array}{lll}
\mathbf{h}_{0}^{T} & \mathbf{h}_{1}^{T} & \mathbf{h}_{2}^{T}
\end{array}\right]^{H}, \mathbf{e}=\left[\begin{array}{lll}
\mathbf{e}_{0}^{T} & \mathbf{e}_{1}^{T} & \mathbf{e}_{2}^{T}
\end{array}\right]^{H} .
\end{aligned}
$$

Accordingly, the expression of $P_{R}$ can be rewritten in the following form

$$
P_{R}=\mathbf{e}^{H} \mathbf{M}_{R} \mathbf{e}+2 \operatorname{Re}\left\{\mathbf{e}^{H} \mathbf{m}_{R}\right\}+a,
$$

where $\quad \mathbf{M}_{R}=\sum_{i=0}^{2} P_{i} \mathbf{F}_{i}, \quad \mathbf{m}_{R}=\sum_{i=0}^{2} P_{i} \mathbf{F}_{i} \mathbf{h}, a=\mathbf{h}^{H} \sum_{i=0}^{2} P_{i} \mathbf{F}_{i} \mathbf{h}+\sigma_{R}^{2} f, \quad \mathbf{F}_{i}=\mathbf{G}_{i 1}^{T}\left(\mathbf{I}_{K^{2} \times K^{2}} \circ \mathbf{S}\right) \mathbf{G}_{i 1}$, $f=\mathbf{1}_{K^{2} \times 1}{ }^{T}\left(\mathbf{I}_{K^{2} \times K^{2}} \circ \mathbf{S}\right) \mathbf{1}_{K^{2} \times 1}$.

Since the CSI errors are much smaller than the estimations of CSI, we neglect all the third and fourth order terms of the CSI errors and the cross-product terms. Thus, the Eq. (8) can be reformulated as

$$
P_{s}^{i}=\mathbf{e}^{H} \mathbf{L}_{i} \mathbf{e}+2 \operatorname{Re}\left\{\mathbf{e}^{H} \mathbf{l}_{i}\right\}+\tau_{i},
$$

where $\mathbf{L}_{i}=P_{\bar{i}} \mathbf{Z}_{i} \mathbf{S} \mathbf{Z}_{i}^{H}, \quad \mathbf{l}_{i}=P_{\bar{i}} \mathbf{Z}_{i} \mathbf{S} \mathbf{g}_{i \bar{i}}^{*}, \quad \tau_{i}=P_{\bar{i}} \mathbf{g}_{i \bar{i}}^{T} \mathbf{S} \mathbf{g}_{i \bar{i}}^{*}, \mathbf{Z}_{i}=\mathbf{G}_{\bar{i} 2}^{T} \mathbf{C}_{i}+\mathbf{G}_{i 1}^{T} \mathbf{D}_{\bar{i}}$.

The mathematical proof of Eq. (16) please refer to the Appendix.

Similarly, Eq. (9), Eq. (10) and Eq. (12) can be expressed, respectively, by the following forms 


$$
\begin{gathered}
P_{\mathrm{int}}{ }^{i}+P_{n}^{i}=\mathbf{e}^{H} \mathbf{N}_{i} \mathbf{e}+2 \operatorname{Re}\left\{\mathbf{e}^{H} \mathbf{n}_{i}\right\}+d_{i}, \\
P_{I}=\mathbf{e}^{H} \mathbf{M}_{0} \mathbf{e}+2 \operatorname{Re}\left\{\mathbf{e}^{H} \mathbf{m}_{0}\right\}+c_{00},
\end{gathered}
$$

where

$$
\begin{aligned}
& \mathbf{N}_{i}=P_{i} \mathbf{T}_{i} \mathbf{S} \mathbf{T}_{i}^{H}+P_{0} \mathbf{V}_{i} \mathbf{S} \mathbf{V}_{i}^{H}+\sigma_{R}^{2} \mathbf{F}_{i}, \\
& \mathbf{n}_{i}=P_{0} \mathbf{V}_{i} \mathbf{S g}_{i 0}^{*}+\sigma_{R}^{2} \mathbf{F}_{i} \mathbf{h}, \\
& d_{i}=P_{0} \mathbf{g}_{i 0}^{T} \mathbf{S g}_{i 0}^{*}+\sigma_{R}^{2} \hat{\mathbf{h}}^{H} \mathbf{F}_{i} \hat{\mathbf{h}}+\sigma_{i}^{2}, \\
& \mathbf{T}_{i}=\mathbf{G}_{i 2}^{T} \mathbf{C}_{i}+\mathbf{G}_{i 1}^{T} \mathbf{D}_{i}, \mathbf{V}_{i}=\mathbf{G}_{01}^{T} \mathbf{C}_{i}+\mathbf{G}_{i i}^{T} \mathbf{D}_{0}, \\
& \mathbf{M}_{0}=\sum_{i=0}^{2} P_{i} \mathbf{U}_{i} \mathbf{S} \mathbf{U}_{i}^{H}+\sigma_{R}^{2} \mathbf{F}_{0}, \\
& \mathbf{m}_{0}=\sum_{i=0}^{2} P_{i} \mathbf{U}_{i} \mathbf{S} \mathbf{g}_{0 i}^{*}+\sigma_{R}^{2} \mathbf{F}_{0} \mathbf{h}, \\
& c_{00}=\sum_{i=0}^{2} P_{i} \mathbf{g}_{0 i}^{T} \mathbf{S g}_{0 i}^{*}+\sigma_{R}^{2} \mathbf{h}^{H} \mathbf{F}_{0} \mathbf{h}, \\
& \mathbf{U}_{i}=\mathbf{G}_{i 1}^{T} \mathbf{C}_{0}+\mathbf{G}_{01}^{T} \mathbf{D}_{i} .
\end{aligned}
$$

Hence, the optimization problem can be reformulated as Problem 2, and given as:

$$
\begin{array}{ll}
\min _{\mathbf{S}, t} & t \\
\text { s.t. } & \operatorname{Pr}\left\{\mathbf{e}^{H} \mathbf{M}_{R} \mathbf{e}+2 \operatorname{Re}\left\{\mathbf{e}^{H} \mathbf{m}_{R}\right\} \geq c_{R}\right\} \geq \rho_{R} \\
& \operatorname{Pr}\left\{\mathbf{e}^{H} \mathbf{M}_{i} \mathbf{e}+2 \operatorname{Re}\left\{\mathbf{e}^{H} \mathbf{m}_{i}\right\} \leq c_{i}\right\} \leq \rho_{i} \quad\{i=1,2\} \\
& \operatorname{Pr}\left\{\mathbf{e}^{H} \mathbf{M}_{0} \mathbf{e}+2 \operatorname{Re}\left\{\mathbf{e}^{H} \mathbf{m}_{0}\right\} \geq c_{0}\right\} \leq \rho_{0} \\
& \operatorname{rank}(\mathbf{S})=1, \mathbf{S} \geq 0,
\end{array}
$$

where $c_{R}=t-a, c_{i}=\gamma_{i} d_{i}-\tau_{i}, c_{0}=\zeta-c_{00}, \mathbf{M}_{i}=\mathbf{L}_{i}-\gamma_{i} \mathbf{N}_{i}, \mathbf{m}_{i}=\mathbf{l}_{i}-\gamma_{i} \mathbf{n}_{i}$. How we can solve this optimization problem is the subject of next section.

\subsection{Robust Beamforming Design Based on Bernstein-Type Inequalities}

In order to solve such non-convex and intractable probabilistic constrained optimization problem, we adopt two kinds of Bernstein-type inequalities, which are widely utilized to cope with the probabilistic constraints [18, 20, 23], then Eq. (13b)-(13d) can be transformed to deterministic forms.

Before utilizing Bernstein-type inequalities, we rewrite the CSI errors as $\mathbf{e}_{i}=\mathbf{E}_{i}^{1 / 2} \mathbf{v}_{i}$, where $\mathbf{E}_{i}^{1 / 2}$ is the positive semidefinite square root of $\mathbf{E}_{i}$ and $\mathbf{v}_{i} \sim \mathcal{C N}\left(\mathbf{0}, \mathbf{I}_{K \times K}\right)$. Accordingly, we have $\mathbf{e}=\mathbf{E}^{1 / 2} \mathbf{v}$, where $\mathbf{E}=\operatorname{BD}\left(\mathbf{E}_{0}, \mathbf{E}_{1}, \mathbf{E}_{2}\right), \mathbf{v}=\left[\mathbf{v}_{0}^{T} \mathbf{v}_{1}^{T} \mathbf{v}_{2}^{T}\right]^{H}$.

Then the probabilistic constraints in Eq. (19b)-(19d) can be reformulated as Eq. (20)-(22) respectively 


$$
\begin{aligned}
& \operatorname{Pr}\left\{\mathbf{v}^{H} \widehat{\mathbf{M}}_{R} \mathbf{v}+2 \operatorname{Re}\left\{\mathbf{v}^{H} \widehat{\mathbf{m}}_{R}\right\} \leq c_{R}\right\} \geq 1-\rho_{R}, \\
& \operatorname{Pr}\left\{\mathbf{v}^{H} \widehat{\mathbf{M}}_{i} \mathbf{v}+2 \operatorname{Re}\left\{\mathbf{v}^{H} \widehat{\mathbf{m}}_{i}\right\} \geq c_{i}\right\} \geq 1-\rho_{i}, \\
& \operatorname{Pr}\left\{\mathbf{v}^{H} \widehat{\mathbf{M}}_{0} \mathbf{v}+2 \operatorname{Re}\left\{\mathbf{v}^{H} \widehat{\mathbf{m}}_{0}\right\} \leq c_{0}\right\} \geq 1-\rho_{0},
\end{aligned}
$$

where $\widehat{\mathbf{M}}_{j}=\mathbf{E}^{1 / 2} \mathbf{M}_{j} \mathbf{E}^{1 / 2}, \widehat{\mathbf{m}}_{j}=\mathbf{E}^{1 / 2} \mathbf{m}_{j}$, and $j \in\{0,1,2, R\}$.

Then the probabilistic constraints in Eq. (20)-(22) can be transformed into tractable forms by introducing the two kinds of Bernstein-type inequalities effectively [26]. The probabilistic constraint of $P_{R}$, SINR and $P_{I}$ can be written as the following deterministic form respectively

$$
\begin{aligned}
& \operatorname{Tr}\left(\widehat{\mathbf{M}}_{R}\right)+\sqrt{2 \delta_{R}} \sqrt{\left\|\operatorname{vec}\left(\hat{\mathbf{M}}_{R}\right)\right\|^{2}+2\left\|\widehat{\mathbf{m}}_{R}\right\|^{2}}+\delta_{R} s^{+}\left(\widehat{\mathbf{M}}_{R}\right) \leq c_{R}, \\
& \operatorname{Tr}\left(\hat{\mathbf{M}}_{i}\right)-\sqrt{2 \delta_{i}} \sqrt{\left\|\operatorname{vec}\left(\hat{\mathbf{M}}_{i}\right)\right\|^{2}+2\left\|\widehat{\mathbf{m}}_{i}\right\|^{2}}-\delta_{i} s^{-}\left(\widehat{\mathbf{M}}_{i}\right) \geq c_{i}, \\
& \operatorname{Tr}\left(\hat{\mathbf{M}}_{0}\right)+\sqrt{2 \delta_{0}} \sqrt{\left\|\operatorname{vec}\left(\hat{\mathbf{M}}_{0}\right)\right\|^{2}+2\left\|\widehat{\mathbf{m}}_{0}\right\|^{2}}+\delta_{0} s^{+}\left(\hat{\mathbf{M}}_{0}\right) \leq c_{0},
\end{aligned}
$$

where $\delta_{R}=-\ln \left(\rho_{R}\right), \delta_{j}=-\ln \left(\rho_{j}\right), \delta_{0}=-\ln \left(\rho_{0}\right)$. Then, Eq. (23)-(25) can be reformulated as the following convex form respectively

$$
\begin{gathered}
\left\{\begin{array}{l}
\operatorname{Tr}\left(\widehat{\mathbf{M}}_{R}\right)+\sqrt{2 \delta_{R}} a_{R}+\delta_{R} b_{R} \leq c_{R} \\
\sqrt{\left\|\operatorname{vec}\left(\hat{\mathbf{M}}_{R}\right)\right\|^{2}+2\left\|\widehat{\mathbf{m}}_{R}\right\|^{2}} \leq a_{R}, \\
b_{R} \mathbf{I}-\widehat{\mathbf{M}}_{R} \geq 0
\end{array}\right. \\
\left\{\begin{array}{l}
\operatorname{Tr}\left(\widehat{\mathbf{M}}_{i}\right)-\sqrt{2 \delta_{i}} a_{i}-\delta_{i} b_{i} \geq c_{i} \\
\sqrt{\left\|\operatorname{vec}\left(\hat{\mathbf{M}}_{i}\right)\right\|^{2}+2\left\|\widehat{\mathbf{m}}_{i}\right\|^{2}} \leq a_{i} \\
b_{i} \mathbf{I}+\hat{\mathbf{M}}_{i} \geq 0
\end{array},\right. \\
\left\{\begin{array}{l}
\operatorname{Tr}\left(\widehat{\mathbf{M}}_{0}\right)+\sqrt{2 \delta_{0}} a_{0}+\delta_{0} b_{0} \leq c_{0} \\
\sqrt{\left\|\operatorname{vec}\left(\hat{\mathbf{M}}_{0}\right)\right\|^{2}+2\left\|\widehat{\mathbf{m}}_{0}\right\|^{2}} \leq a_{0} \\
b_{0} \mathbf{I}-\widehat{\mathbf{M}}_{0} \geq 0
\end{array},\right.
\end{gathered}
$$

where $a_{R}, b_{R}, a_{i}, b_{i}, a_{0}$ and $b_{0}$ are slack variables, and $b_{R} \geq 0, b_{i} \geq 0, b_{0} \geq 0$. 
Accordingly, the problem 2 can be conservatively transformed as Problem 3:

$$
\begin{array}{cl}
\min _{\mathbf{S}, t} & t \\
\text { s.t. } & (26), \quad(27), \quad(28) \\
& \operatorname{rank}(\mathbf{S})=1, \mathbf{S} \geq 0 \\
& b_{R} \geq 0, b_{i} \geq 0, \quad b_{0} \geq 0, \quad \forall i \in\{1,2\}
\end{array}
$$

However, the optimization problem in Eq. (29) is still non-convex due to the rank-one constraint. Fortunately, we can convert the Problem 3 into a convex SDP problem by using semi-definite relaxation (SDR) technique, which can be efficiently solved by the well-known SDP solvers such as CVX [27]. Then we can derive the optimal solution $\mathbf{S}_{\text {opt }}$. If $\operatorname{rank}\left(\mathbf{S}_{\text {opt }}\right)=1$, the optimal solution of Problem 1 can be acquired by adopting eigenvalue decomposition. When $\operatorname{rank}\left(\mathbf{S}_{\text {opt }}\right)>1$, randomization and eigenvector approximation can be used to derive the approximate solution [28] .

\subsection{Robust Beamforming Design Based on S-Procedure}

For comparison purpose, in this subsection we cope with the probabilistic constrained Problem 2 by applying an extended S-Procedure. In order to use this scheme, the statistical distribution CSI errors model will be replaced by the bounded model [3]. We can obtain the safe approximation of the probabilistic constraints of Eq. (19b)-(19d) as

$$
\begin{aligned}
& \mathbf{e}^{H} \mathbf{M}_{R} \mathbf{e}+2 \operatorname{Re}\left\{\mathbf{e}^{H} \mathbf{m}_{R}\right\}-C_{R} \leq 0, \\
& \mathbf{e}^{H} \mathbf{M}_{i} \mathbf{e}+2 \operatorname{Re}\left\{\mathbf{e}^{H} \mathbf{m}_{i}\right\}-C_{i} \geq 0, \\
& \mathbf{e}^{H} \mathbf{M}_{0} \mathbf{e}+2 \operatorname{Re}\left\{\mathbf{e}^{H} \mathbf{m}_{0}\right\}-c_{0} \leq 0,
\end{aligned}
$$

while e in Eq. (30)-(32) satisfy the following constraints

$$
\operatorname{Pr}\left\{\mathbf{e}_{i}^{H} \mathbf{e}_{i} \leq r_{i}^{2}\right\} \geq 1-\rho_{i}, \quad i \in\{0,1,2\},
$$

where $r_{i}$ is the radius of the sphere. It is easy to see that $r_{i}^{2}$ is a Chi-square random variable with $2 K$ degrees of freedom. It can be calculated as [29]

$$
r_{i}^{2}=\Phi_{\chi_{2 K}^{2}}^{-1}\left(1-\rho_{i}\right) \varepsilon / 2, \quad i \in\{0,1,2\}
$$

where $\Phi_{\chi_{2 K}^{2}}^{-1}(x)$ is the inverse cumulative distribution function of an Chi-square random variable with $2 K$ degrees of freedom at $x$. Currently, the optimization problem is still difficult to be solved due to the CSI error vectors are continuous over the given sphere error bounding, so the constraint is semi-infinite. Then, we transform the semi-infinite constraints to a finite number of LMIs by the extended S-procedure [30]. 
Based on the S-procedure, the constraints in Eq. (19b)-(19d) can be converted to Eq. (35)-(37) respectively

$$
\begin{gathered}
{\left[\begin{array}{cc}
-\mathbf{M}_{R}+\sum_{j=0}^{2} \lambda_{R j} \mathbf{A}_{j}^{H} \mathbf{A}_{j} & -\mathbf{m}_{R} \\
-\mathbf{m}_{R}{ }^{H} & c_{R}-\sum_{j=0}^{2} \lambda_{R j} r_{j}^{2}
\end{array}\right] \geq 0,} \\
{\left[\begin{array}{cc}
\mathbf{M}_{i}+\sum_{j=0}^{2} \lambda_{i j} \mathbf{A}_{j}^{H} \mathbf{A}_{j} & \mathbf{m}_{i} \\
\mathbf{m}_{i}^{H} & -c_{i}-\sum_{j=0}^{2} \lambda_{i j} r_{i}^{2}
\end{array}\right] \geq 0,} \\
{\left[\begin{array}{cc}
-\mathbf{M}_{0}+\sum_{j=0}^{2} \lambda_{0 j} \mathbf{A}_{j}^{H} \mathbf{A}_{j} & -\mathbf{m}_{0} \\
-\mathbf{m}_{0}^{H} & c_{0}-\sum_{j=0}^{2} \lambda_{0 j} r_{0}^{2}
\end{array}\right] \geq 0,}
\end{gathered}
$$

where $\lambda_{R j} \geq 0, \lambda_{i j} \geq 0, \lambda_{0 j} \geq 0$ are slack variables.

Then Problem 2, using the above equivalent constraints, can be reformulated to Problem 4:

$$
\begin{array}{cl}
\min _{\mathbf{S}, t} & t \\
\text { s.t. } & (35) \quad(36) \quad(37) \\
& \operatorname{rank}(\mathbf{S})=1, \mathbf{S} \geq 0 \\
& \lambda_{R j} \geq 0, \lambda_{i j} \geq 0, \lambda_{0 j} \geq 0 \\
& \forall i \in\{1,2\}, \forall j \in\{0,1,2\}
\end{array}
$$

The optimization problem in Eq.(38) can be solved by CVX after dropping the rank-one constraint. And moreover, we provide the computational complexity of problems in Eq. (29), Eq. (38) after dropping the rank-one constraint. The computational complexity is main from the computation of SDP. Problem 3 in Eq. (29) involves 4 linear matrix inequality (LMI) constraints of size $3 K$, 4 second-order cone (SOC) constraints and 8 LMI constraints of size 1. While problem 4 in Eq. (38) has 4 LMI constraints of size $3 K+1$ and 12 LMI constraints of size 1 . In addition, both of the problems have $1 \mathrm{LMI}$ constraints of size $K^{2}$. Thus, problems 3 based Bernstein-type inequalities has higher computational cost due to the more complicated constraints [31]. 


\section{Simulation Results and Discussions}

In the following simulations, the channels are set to be Rayleigh flat fading environment, and the channel coefficients are $\mathbf{h}_{0}=\mathcal{C N}(\mathbf{0}, 0.5 \times \mathbf{I}), \mathbf{h}_{1}=\mathbf{h}_{2}=\mathcal{C N}(\mathbf{0}, \mathbf{I})$. The number of relays is $K=4$. The transmit power of PU and $S_{i}$ are $P_{0}=P_{1}=P_{2}=8 \mathrm{dBw}$. The outage probabilities are all set to be 0.1, i.e. $\rho_{0}=\rho_{1}=\rho_{2}=0.1$. The noise variances at all receivers are assumed to be equal to 1 , i.e. $\sigma_{1}^{2}=\sigma_{2}^{2}=\sigma_{R}^{2}=1$. The CSI errors' variance is set $\varepsilon=0.002$. The target SINR threshold at the two SUs are assumed to be the same, i.e. $\gamma_{1}=\gamma_{2}=\gamma$.

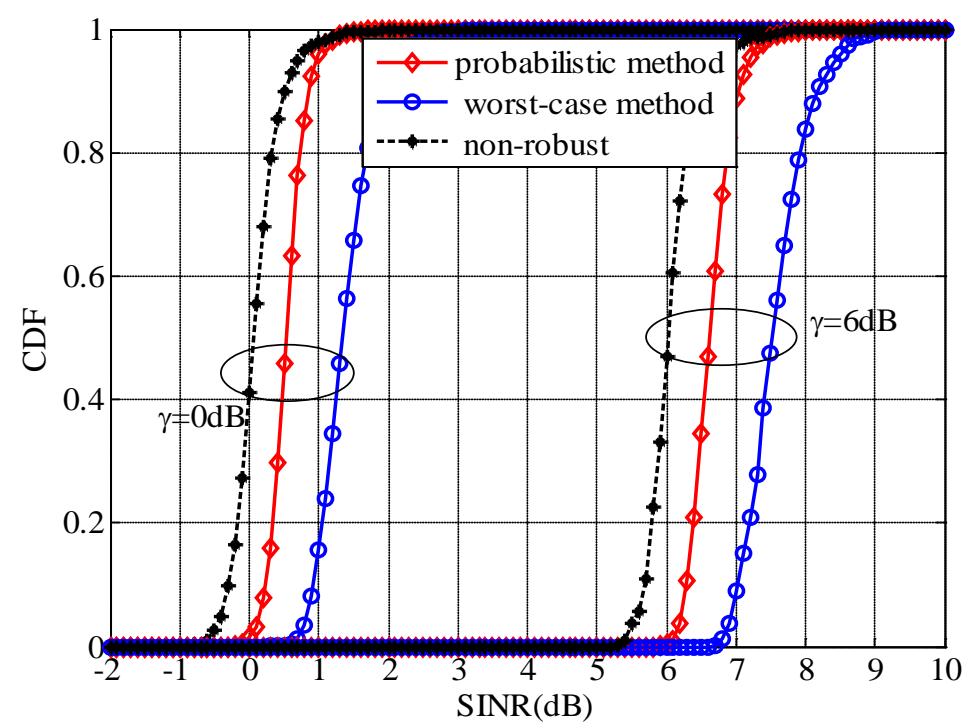

Fig. 2. The CDF of SINR at secondary user for different value of SINR threshod ( $\gamma=0 \mathrm{~dB}, \gamma=6 \mathrm{~dB}$ ).

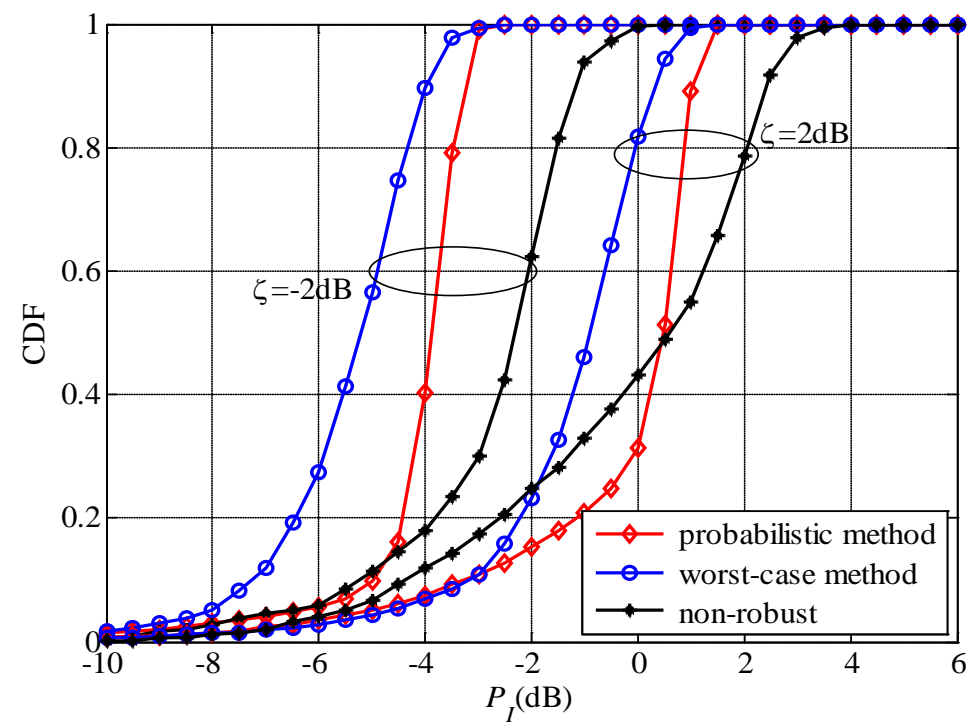

Fig. 3. The CDF of interference power at PU for different values of interference power threshold $(\zeta=2 \mathrm{~dB}, \zeta=-2 \mathrm{~dB})$. 
In Fig. 2 and Fig. 3, we illustrate the cumulative distribution function (CDF) of SINR at SU and the interference power at PU over 2000 random channel realizations respectively. The robust beamforming designs based on Bernstein-type inequalities and S-Procedure, and non-robust beamforming design are compared. In Fig. 2, we set interference threshold at PU to be $\zeta=0 \mathrm{~dB}$, it can be obviously seen that the SINR of the non-robust beamforming design cannot always satisfy the given SINR threshold, more specifically, more than $50 \%$ cannot guarantee the target SINR value, i.e. $\gamma=0 \mathrm{~dB}$ and $\gamma=6 \mathrm{~dB}$. For both robust beamforming designs, less than $10 \%$ of the achieved SINR values cannot satisfy the the target SINR thresholds. That is to say, the robust beamforming designs can always guarantee probabilistic constraint of target SINR at each SU. In Fig. 3, we set SINR thresholds at $S_{1}$ and $S_{2}$ to be the same, i.e. $\gamma=8 \mathrm{~dB}$. We compare the CDF of the interference power at PU between the proposed robust beamforming design and non-robust beamforming design versus different interference thresholds. It is clear that the non-robust design performs worst, about $20 \%$ of the interference power exceeds the interference threshold when $\zeta=2 \mathrm{~dB}$, and nearly $40 \%$ of the interference power exceeds the interference threshold when $\zeta=-2 \mathrm{~dB}$, while both the proposed probabilistic method and the worst-case method robust beamforming design can guarantee the predetermined interference threshold. That is to say, from Fig. 2 and Fig. 3, it can be verified that the proposed probabilistic method and worst-case method are both robust to the CSI errors.



Fig. 4. Feasibility rate (\%) versus target SINR $\gamma(\mathrm{dB})$. 


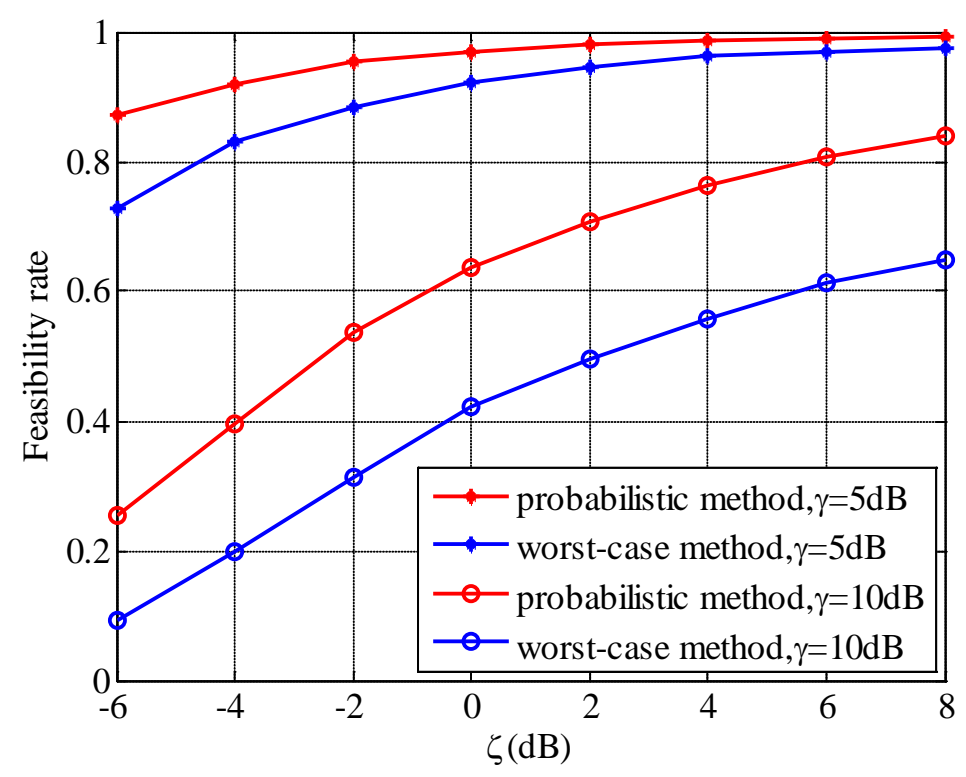

Fig. 5. Feasibility rate (\%) versus interference threshold $\zeta(\mathrm{dB})$.

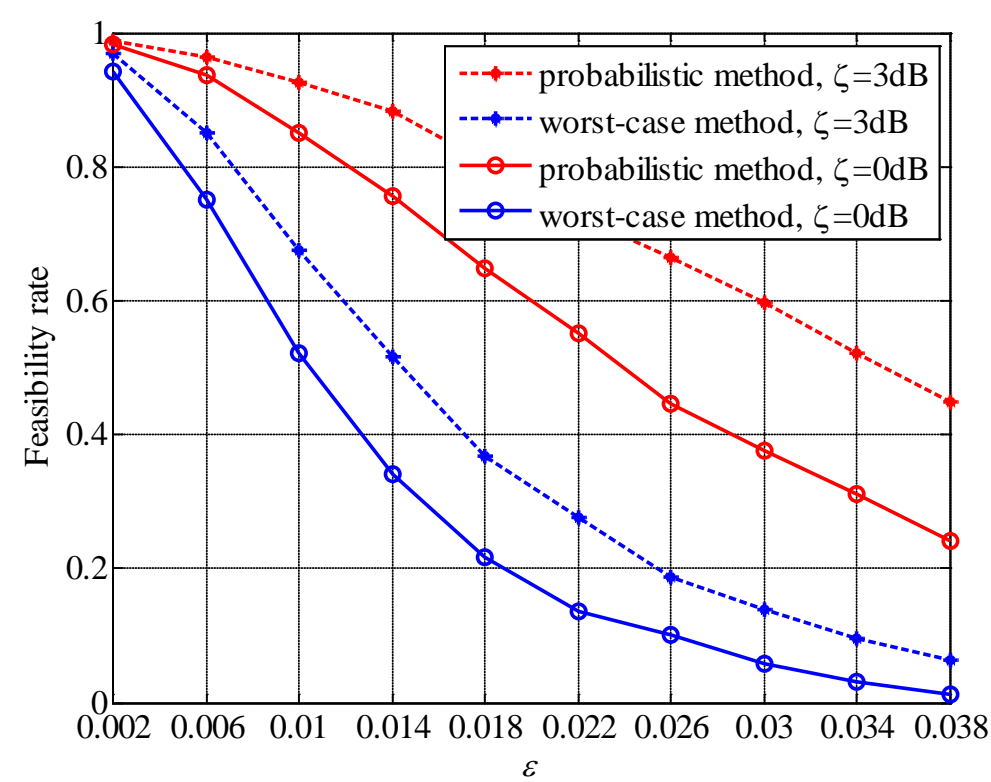

Fig. 6. Feasibility rate (\%) versus the CSI error variance $\varepsilon$ for different interference threshold $\zeta(\mathrm{dB})$.

In Fig. 4, Fig. 5 and Fig. 6, we plot the feasibility rates of the two robust beamforming designs versus the target SINR $\gamma$, the interference threshold $\zeta$ and the CSI error variance $\varepsilon$, respectively. In Fig. 4, we compare the feasibility rates of the two proposed robust beamforming design versus the target SINRs with $K=4$ and $K=5$. As expected, the feasibility rates decrease with the increase of target SINR $\gamma$. It is also shown that the feasibility rates increase corresponding with the increase of the number of relay. In addition, for a given target SINR value and interference threshold, the feasibility rate of robust 
beamforming design based on probabilistic method is always higher than the worst-case method invariably. Fig. 5 plots the feasibility rates of the of the two robust beamforming designs versus the interference threshold with different target SINR values, i.e. $\gamma=5 \mathrm{~dB}$ and $\gamma=10 \mathrm{~dB}$. It is shown that the feasibility rate decreases with the increase of target SINR value for a certain interference threshold as expected, and increases with the interference threshold increases. Moreover, we can observe that the probabilistic method based on probabilistic method can support higher region of interference threshold compared with the worst-case method. In Fig. 6, we illustrate the feasibility rates of the two robust beamforming designs versus the CSI error variance $\varepsilon$ for different values of interference threshold with a certain target SINR value, i.e. $\gamma=4 \mathrm{~dB}$. We can see that the feasibility rates of the both methods decrease with the increase of $\varepsilon$, and the feasibility rate of the probabilistic method is always higher than the worst-case method for different values of interference threshold. In a word, from Fig. 4, Fig. 5 and Fig. 6, it can be verified that the probabilistic method can provide higher feasibility rate than the worst-case method.

In Fig. 7, we plot the minimum transmit power budget of the two proposed robust beamforming designs versuses the target SINR $\gamma$, the transmit power with perfect CSI is also presented. It is shown that the transmit power budget of relay decrease with the increase of $K$. We also see that the higher target SINR require more transmit power of relays, and the problem is infeasible for a critical SINR threthod. And the probabilistic method based on Bernstein-type inequalities require less power than the worst-case method. That is to say, the probabilistic method is much more energy-saving.

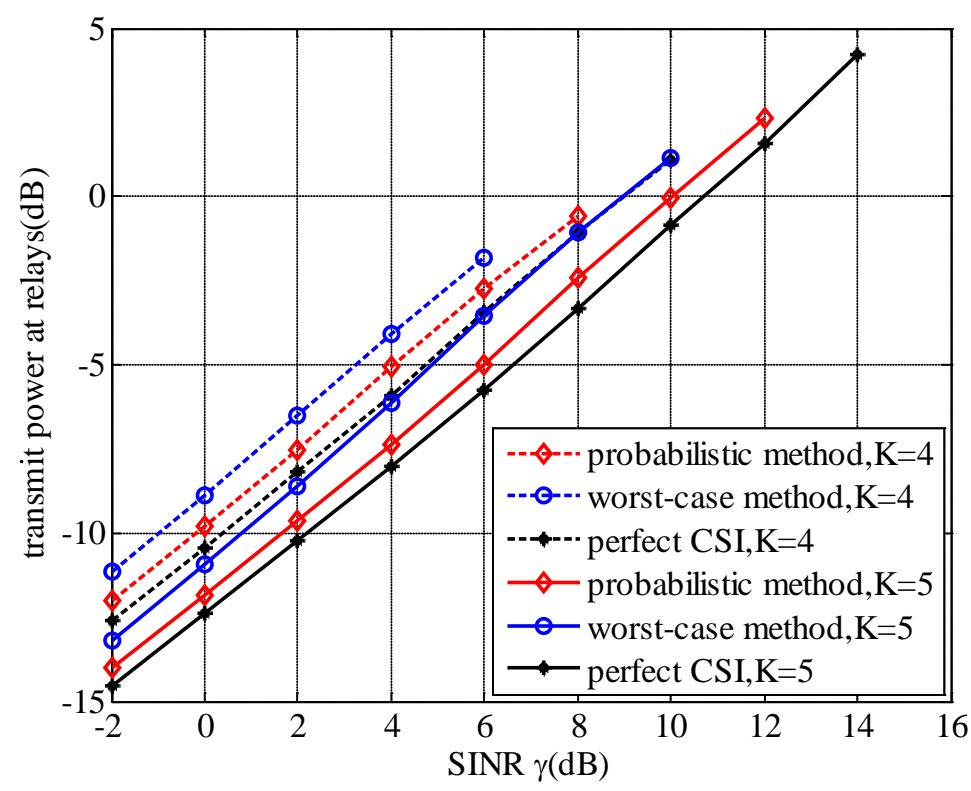

Fig. 7. Total transmit power $(\mathrm{dB})$ versus target SINR $\gamma(\mathrm{dB})$.

\section{Conclusions}

This paper investigates the distributed robust beamforming design with the goal of minimizing the total transmit power of collaborative relay with probabilistic constraints in cognitive two-way relay networks. The CSI errors model is a known circular complex Gaussian random 
distribution in the system. After introducing an auxiliary variable and a series of matrix transformations, we employ the probabilistic method based on two kinds of Bernstein-type inequalities and worst-case method based on extended S-procedure to tackle the original non-convex optimization problem respectively. Simulation results demonstrate that the both robust beamforming designs can overcome the effects of CSI errors. Moreover, the probabilistic method based on Bernstein-type inequalities is more promising than the worst-case method, since it can make trade-off between robustness and effectiveness.

\section{Appendix}

To prove Eq. (16), we have

$$
\hat{\mathbf{h}}_{i}^{T} \mathbf{W} \hat{\mathbf{h}}_{\bar{i}}=\left[\left(\hat{\mathbf{h}}_{\bar{i}} \otimes \mathbf{1}\right) \circ\left(\mathbf{1} \otimes \hat{\mathbf{h}}_{i}\right)\right]^{T} \operatorname{vec}(\mathbf{W})=\mathbf{g}_{i \bar{i}}^{T} \mathbf{u},
$$

where $\mathbf{g}_{i j}=\left(\hat{\mathbf{h}}_{j} \otimes \mathbf{1}_{K \times 1}\right) \circ\left(\mathbf{1}_{K \times 1} \otimes \hat{\mathbf{h}}_{i}\right), \mathbf{u}=\operatorname{vec}(\mathbf{W})$.

Since $\left(\mathbf{e}_{\bar{i}} \otimes \mathbf{1}\right) \circ\left(\mathbf{1} \otimes \hat{\mathbf{h}}_{i}\right)=\operatorname{diag}\left(\mathbf{1} \otimes \hat{\mathbf{h}}_{i}\right)\left(\mathbf{e}_{\bar{i}} \otimes \mathbf{1}\right), \quad\left(\mathbf{e}_{\bar{i}} \otimes \mathbf{1}\right)^{T}=\mathbf{e}^{H} \mathbf{G}_{\bar{i} 2}^{T}, \quad \mathbf{C}_{i}=\operatorname{diag}\left(\mathbf{1}_{K \times 1} \otimes \hat{\mathbf{h}}_{i}\right)$, it can be proved that

$$
\begin{aligned}
\hat{\mathbf{h}}_{i}^{T} \mathbf{W} \mathbf{e}_{\bar{i}} & =\left[\left(\mathbf{e}_{\bar{i}} \otimes \mathbf{1}\right) \circ\left(\mathbf{1} \otimes \hat{\mathbf{h}}_{i}\right)\right]^{T} \operatorname{vec}(\mathbf{W}) \\
& =\left[\operatorname{diag}\left(\mathbf{1} \otimes \hat{\mathbf{h}}_{i}\right)\left(\mathbf{e}_{\bar{i}} \otimes \mathbf{1}\right)\right]^{T} \mathbf{u} . \\
& =\left(\mathbf{e}_{\bar{i}} \otimes \mathbf{1}\right)^{T} \operatorname{diag}\left(\mathbf{1} \otimes \hat{\mathbf{h}}_{i}\right) \mathbf{u} \\
& =\mathbf{e}^{H} \mathbf{G}_{\bar{i} 2}^{T} \mathbf{C}_{i} \mathbf{u}
\end{aligned} .
$$

Similarly, we have

$$
\begin{aligned}
\mathbf{e}_{i}^{T} \mathbf{W} \hat{\mathbf{h}}_{\bar{i}} & =\left[\left(\hat{\mathbf{h}}_{\bar{i}} \otimes \mathbf{1}\right) \circ\left(\mathbf{1} \otimes \mathbf{e}_{i}\right)\right]^{T} \operatorname{vec}(\mathbf{W}) \\
& =\left[\operatorname{diag}\left(\hat{\mathbf{h}}_{\bar{i}} \otimes \mathbf{1}\right)\left(\mathbf{1} \otimes \mathbf{e}_{i}\right)\right]^{T} \mathbf{u} \\
& =\left(\mathbf{1} \otimes \mathbf{e}_{i}\right)^{T} \operatorname{diag}\left(\hat{\mathbf{h}}_{\bar{i}} \otimes \mathbf{1}\right) \mathbf{u} \\
& =\mathbf{e}^{H} \mathbf{G}_{i 1}^{T} \mathbf{D}_{\bar{i}} \mathbf{u}
\end{aligned}
$$

Since in practice, the estimation errors $\mathbf{e}_{i}$ and $\mathbf{e}_{\bar{i}}$ are much smaller than the channel estimates $\mathbf{h}_{i}$ and $\mathbf{h}_{\bar{i}}$, the third and the fourth order terms of $\mathbf{e}_{i}$ and $\mathbf{e}_{\bar{i}}$, and their cross-product terms can be considered to be negligible. Then the Eq. (8) can be expressed as 


$$
\begin{aligned}
P_{s}^{i} & =P_{\bar{i}}\left\|\left(\hat{\mathbf{h}}_{i}+\mathbf{e}_{i}\right)^{T} \mathbf{W}\left(\hat{\mathbf{h}}_{\bar{i}}+\mathbf{e}_{\bar{i}}\right)\right\|^{2} \\
& =P_{\bar{i}}\left\|\hat{\mathbf{h}}_{i}^{T} \mathbf{W} \hat{\mathbf{h}}_{\bar{i}}+\hat{\mathbf{h}}_{i}^{T} \mathbf{W} \mathbf{e}_{\bar{i}}+\mathbf{e}_{i}^{T} \mathbf{W} \hat{\mathbf{h}}_{\bar{i}}\right\|^{2} \\
& =P_{\bar{i}}\left\|\mathbf{g}_{i \bar{i}}^{T} \mathbf{u}+\mathbf{e}^{H} \mathbf{G}_{\bar{i} 2}^{T} \mathbf{C}_{i} \mathbf{u}+\mathbf{e}^{H} \mathbf{G}_{i i}^{T} \mathbf{D}_{\bar{i}} \mathbf{u}\right\|^{2} \\
& =P_{\bar{i}}\left\|\mathbf{g}_{i i}^{T} \mathbf{u}+\mathbf{e}^{H} \mathbf{Z}_{i} \mathbf{u}\right\|^{2} \\
& =\mathbf{e}^{H} P_{\bar{i}} \mathbf{Z}_{i} \mathbf{S} \mathbf{Z}_{i}^{H} \mathbf{e}+2 \operatorname{Re}\left\{\mathbf{e}^{H} P_{\bar{i}} \mathbf{Z}_{i} \mathbf{S} \mathbf{g}_{i \bar{i}}^{*}\right\}+P_{\bar{i}} \mathbf{g}_{i \bar{i}}^{T} \mathbf{S} \mathbf{g}_{i \bar{i}}^{*}
\end{aligned}
$$

Define $\mathbf{L}_{i}=P_{\bar{i}} \mathbf{Z}_{i} \mathbf{S} \mathbf{Z}_{i}^{H}, \mathbf{l}_{i}=P_{\bar{i}} \mathbf{Z}_{i} \mathbf{S g}_{i \bar{i}}^{*}$ and $\tau_{i}=P_{\bar{i}} \mathbf{g}_{i \bar{i}}^{T} \mathbf{S g}_{i \bar{i}}^{*}$, then we have

$$
P_{s}^{i}=\mathbf{e}^{H} \mathbf{L}_{i} \mathbf{e}+2 \operatorname{Re}\left\{\mathbf{e}^{H} \mathbf{I}_{i}\right\}+\tau_{i}
$$

Therefore, we have proved Eq. (16).

\section{References}

[1] J. Mitola and G. Q. Maguire, “Cognitive radio: making software radios more personal,” IEEE Personal Communications, vol. 6, no. 4, pp. 13-18, Aug. 1999. Article (CrossRef Link).

[2] A. Goldsmith, S. A. Jafar, I. Maric, and S. Srinivasa, "Breaking spectrum gridlock with cognitive radios: an information theoretic perspective,” Proceedings of the IEEE, vol. 97, no. 5, pp. 894-914, May. 2009. Article (CrossRef Link).

[3] G. Zheng, K. K. Wong, A. Paulraj, and B. Ottersten, "Robust collaborative-relay beamforming," IEEE Transactions on Signal Processing, vol. 57, no. 8, pp. 3130-3143, Aug. 2009. Article (CrossRef Link).

[4] G. Zheng, K. K. Wong, A. Paulraj, and B. Ottersten, "Collaborative-relay beamforming with perfect CSI: optimum and distributed implementation,” IEEE Signal Processing Letters, vol. 16, no. 4, pp. 257-260, Apr. 2009. Article (CrossRef Link).

[5] D. Zheng, J. Liu, K. K. Wong, H. Chen, and L. Chen, "Robust peer-to-peer collaborative-relay beamforming with ellipsoidal CSI uncertainties,” IEEE Communications Letters, vol. 16, no. 4, pp. 442-445, Apr. 2012. Article (CrossRef Link).

[6] S. S. Ikki and S. Aissa, "Two-way amplify-and-forward relaying with Gaussian imperfect channel estimations,” IEEE Communications Letters, vol. 16, no. 7, pp. 956-959, Jul. 2012. Article (CrossRef Link).

[7] M. Chen and A. Yener, "Multiuser two-way relaying: detection and interference management strategies,” IEEE Transactions on Wireless Communications, vol. 8, no. 8, pp. 4296-4305, Aug. 2009. Article (CrossRef Link).

[8] S. H. Safavi, R. A. S. Zadeh, V. Jamali and S. Salari, "Interference minimization approach for distributed beamforming in cognitive two-way relay networks," in Proc. of 2011 IEEE Pacific Rim Conference on Communications, Computers and Signal Processing, pp. 532-536, Aug. 2011. Article (CrossRef Link).

[9] K. Jitvanichphaibool, Y. C. Liang, and R. Zhang, "Beamforming and power control for multi-antenna cognitive two-way relaying,” in Proc. of 2009 IEEE Wireless Communications and Networking Conference, pp. 1-6, Apr. 2009. Article (CrossRef Link).

[10] A. Afana, A. Ghrayeb, V. Asghari, and S. Affes, "Distributed beamforming for spectrum-sharing systems with AF cooperative two-way relaying,” IEEE Transactions on Communications, vol. 62, no. 9, pp. 3180-3195, Sept. 2014. Article (CrossRef Link). 
[11] S. H. Safavi, M. Ardebilipour and S. Salari, "Relay beamforming in cognitive two-way networks with imperfect channel state information,” IEEE Wireless Communications Letters, vol. 1, no. 4, pp. 344-347, Aug. 2012. Article (CrossRef Link).

[12] P. J. Smith, P. A. Dmochowski, H. A. Suraweera and M. Shafi, "The effects of limited channel knowledge on cognitive radio system capacity,” IEEE Transactions on Vehicular Technology, vol. 62, no. 2, pp. 927-933, Feb. 2013. Article (CrossRef Link).

[13] H. A. Suraweera, P. J. Smith and M. Shafi, "Capacity limits and performance analysis of cognitive radio with imperfect channel knowledge,” IEEE Transactions on Vehicular Technology, vol. 59, no. 4, pp. 1811-1822, May. 2010. Article (CrossRef Link).

[14] M. Tao and R. Wang, "Robust relay beamforming for two-way relay networks," IEEE Communications Letters, vol. 16, no. 7, pp. 1052-1055, Jul. 2012. Article (CrossRef Link).

[15] P. Ubaidulla and S. Aïssa, "Robust distributed two-way relay beamforming in cognitive radio networks," in Proc. of 2012 19th International Conference on Telecommunications (ICT), pp. 1-6, Apr. 2012. Article (CrossRef Link).

[16] S. Ma, Z. L. Dong, J. Ma, and D. Sun, "Robust relay beamforming in cognitive two-way relay networks,” IEEE Communications Letters, vol. 17, no. 10, pp. 2000-2003, Oct. 2013. Article (CrossRef Link).

[17] P. J. Chung, H. Du and J. Gondzio, "A probabilistic constraint approach for robust transmit beamforming with imperfect channel information,” IEEE Transactions on Signal Processing, vol. 59, no. 6, pp. 2773-2782, Jun. 2011. Article (CrossRef Link).

[18] K. Y. Wang, T. H. Chang, W. K. Ma, A. M. C. So and C. Y. Chi, "Probabilistic SINR constrained robust transmit beamforming: a Bernstein-type inequality based conservative approach,” in Proc. of 2011 IEEE International Conference on Acoustics, Speech and Signal Processing (ICASSP), pp. 3080-3083, May. 2011. Article (CrossRef Link).

[19] K. Y. Wang, T. H. Chang, W. K. Ma and C. Y. Chi, “A semidefinite relaxation based conservative approach to robust transmit beamforming with probabilistic SINR constraints,” in Proc. of 2010 18th European Signal Processing Conference, pp. 407-411, Aug. 2010. Article (CrossRef Link)

[20] S. Ma and D. Sun, “Chance constrained robust beamforming in cognitive radio networks," IEEE Communications Letters, vol. 17, no. 1, pp. 67-70, Jan. 2013. Article (CrossRef Link).

[21] C. Xing and et al, "How to understand linear minimum mean-square-error transceiver design for multiple-input-multiple-output systems from quadratic matrix programming," IET Communications, vol. 7, no. 12, pp. 1231-1242, Aug. 2013. Article (CrossRef Link).

[22] C. Xing, S. Ma and Y. Zhou, "Matrix-monotonic optimization for MIMO systems," IEEE Transactions on Signal Processing, vol. 63, no. 2, pp. 334-348, Jan. 2015. Article (CrossRef Link).

[23] D. Ponukumati, F. Gao, and C. Xing, "Robust peer-to-peer relay beamforming: a probabilistic approach,” IEEE Communications Letters, vol. 17, no. 2, pp. 305-308, Feb. 2013. Article (CrossRef Link).

[24] S. Ma, A. M. C. So and K. Yang, "Robust beamforming in two-way relay networks: quartically perturbed chance constrained formulation and tractable approximation," in Proc. of 2014 IEEE International Conference on Acoustics, Speech and Signal Processing (ICASSP), pp. 2734-2738, May. 2014. Article (CrossRef Link).

[25] M. A. Maleki, S. Mehrizi and M. Ahmadian, "Distributed robust beamforming in multi-user relay network," 2014 IEEE Wireless Communications and Networking Conference (WCNC), pp. 904-907, Nov. 2014. Article (CrossRef Link).

[26] I. Bechar, "A Bernstein-type inequality for stochastic processes of quadratic forms of Gaussian variables,” [Online]: http://arxiv.org/abs/0909.3595/.

[27] M. Grant and S.P. Boyd, "CVX: MATLAB software for disciplined convex programming." [Online]: http://cvxr.com/cvx.

[28] Y. Huang and S. Zhang, "Complex matrix decomposition and quadratic programming," Mathematics of Operations Research, vol. 32, no. 3, pp. 758-768, Aug. 2007. Article (CrossRef Link). 
[29] J. Xiong, D. Ma, K. K. Wong, and J. Wei, "Robust masked beamforming for MISO cognitive radio networks with unknown eavesdroppers," IEEE Transactions on Vehicular Technology, vol. 65, no. 2, pp. 744-755, Feb. 2016. Article (CrossRef Link).

[30] S. Boyd and L. Vandenberghe, "Convex Optimization," Cambridge University Press, 2004. Article (CrossRef Link)

[31] I. Pólik and T. Terlaky, "Interior point methods for nonlinear optimization," Nonlinear Optimization, pp. 215-276, Jan. 2010. Article (CrossRef Link)

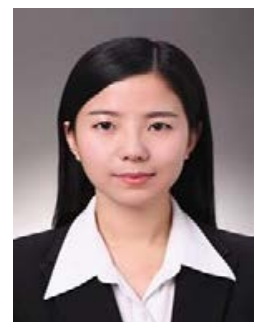

Xueyan Chen received her B.S. degree in Electronic and Information Engineering from PLA Information Engineering University in 2009 and her M.S. degree in Signal and Information Processing from Shenyang Ligong University in 2012. She is currently a Ph.D. student in Signal and Information Processing at BUPT. Her research interests include cognitive radio, relay systems, physical layer security, and energy harvesting.

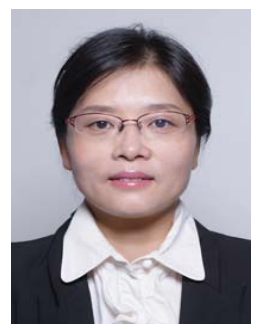

Li Guo was born in 1968, and she is the director of the Nation Engineering Education Center and the director of the Beijing Outside-University Training Center at BUPT. She is also a professor and supervisor of graduate students at the school of Information and Communication Engineering at BUPT. Her major research interests include future wireless communication, mobile cloud computing and data mining based on mobile Internet embedded system design.

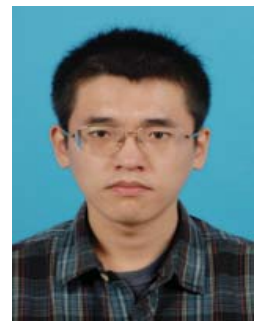

Chao Dong received his B.S and Ph.D. degrees in Signal and Information Processing from the Beijing University of Posts and Telecommunications (BUPT), Beijing, China, in 2007 and 2012, respectively. Since August 2014, he has been a lecturer in the school of Information and Communication Engineering at BUPT. His research interests are mainly on MIMO signal processing, multiuser precoding, decision feedback equalizer and relay signal processing. 


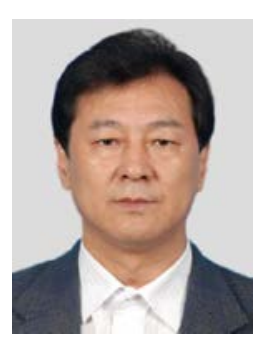

Jiaru Lin received his B.S. and Ph.D. degrees from the School of Information Engineering at BUPT, China, in 1987 and 2001, respectively. From 1991 to 1994, he studied at the Swiss Federal Institute of Technology Zurich. He is now a professor and Ph.D. supervisor in the school of Information and Communication Engineering at BUPT. His research interests include wireless communication, personal communication and communication networks.

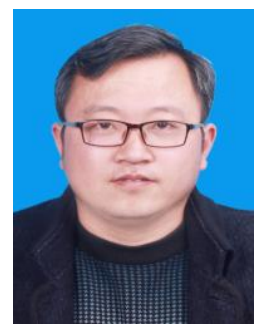

Xingwang $\mathbf{L i}$ received the B.Sc. degree in communication engineering from Henan Polytechnic University, China, in 2007. He then received the M. Sc. degree from the National Key Laboratory of Science and Technology on Communications at University of Electronic Science and Technology of China (UESTC) and Ph. D. degrees in communication and information system from the State Key Laboratory of Networking and Switching Technology at Beijing University of Posts and Telecommunications (BUPT). He is currently a lecturer with the College of Computer Science and Technology, Henan Polytechnic University, Jiaozuo China. His research interests include massive MIMO, hardware constrained communication, FSO communications, and performance analysis of fading channels.

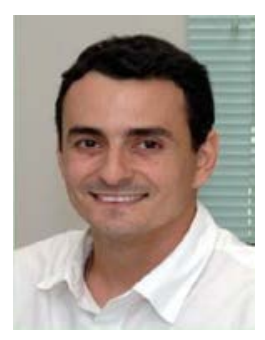

Charles Casimiro Cavalcante received the B.Sc and M.Sc in Electrical Engineering from the Federal University of Cear (UFC), Brazil, in 1999 and 2001, respectively, and the Ph.D. degree from the University of Campinas (UNICAMP), Brazil, in 2004. He has held a grant for Scientific and Technological Development from 2004 to 2007 and since March 2009 he has a grant of Scientific Research Productivity both from the Brazilian Research Council (CNPq). From March 2007 to November 2008 he was a Visiting Professor at Teleinformatics Engineering Department of UFC and since November 2008 he is an Assistant Professor at the same department and university holding the Statistical Signal Processing chair. From August 2014 to July 2015 he was a Visiting Assistent Professor at the Department of Computer Science and Electrical Engineering (CSEE) from University of Maryland, Baltimore County (UMBC) in the United States. He has been working on signal processing strategies for communications where he has several papers published in journal and conferences, has authored three international patents and he has worked on several funded research projects on the signal processing and wireless communications areas. He is also a co-author of the book Unsupervised Signal Processing: Channel Equalization and Source Separation, published by CRC Press. He is a researcher of the Wireless Telecommunications Research Group (GTEL) where he leads research on signal processing and wireless communications. Dr. Cavalcante is a Senior Member of the IEEE and Senior Member of the Brazilian Telecommunications Society (SBrT). His main research interests are in signal processing for communications, blind source separation, wireless communications, and statistical signal processing. 